\section{Venom activity}

Natural venoms are high potency toxins with complex mechanisms of action. In The Journal of Experimental Medicine, Bourgeois et al. show that the human $\mathrm{T}$ cell repertoire contains $\mathrm{T}$ cell clones that react to bee and wasp venom in a manner dependent on the antigen-presenting molecule CD1a. Skin dendritic cells and Langerhans cells, which have high CD1a expression, can prime these responses. Venom fractionation indicates that the venom does not directly provide a lipid antigen but that venom-derived phospholipases such as PLA2 cleave intact, non-antigenic phospholipids into antigenic fatty acids, which activate CD1areactive T cells. Injection of wasp or bee venom (at concentrations that mimic a sting) changes the lipid content of the skin to generate fatty acids that can be predicted by the enzymatic specificity of PLA2, and neutralization of PLA2 blocks CD1aspecific $T$ cell reactivity. This indicates that PLA2 is the essential venom compound for the generation of CD1a ligands.

J. Exp. Med. (12 January 2015) doi:10.1084/jem.20141505

\section{Dopamine controls NLRP3}

Dopamine receptors are expressed on cells of the immune system, and lack of dopamine has been linked to inflammation in the central nervous system. In Cell, Zhou and colleagues report that dopamine negatively regulates the activation of NLRP3 inflammasomes and thereby prevents the processing and release of interleukin $1 \beta$ (IL-1 $\beta$ ) and IL-18. Dopamine has no effect on AIM2 or NLRC4 inflammasomes. Knockdown or deficiency of the dopamine receptor DRD1 abrogates the dopamine-mediated inhibition of NLRP3; conversely, DRD1 agonists also suppress the activation of NLRP3. Mechanistically, dopamineDRD1 elicits the production of CAMP, which promotes Lys48-linked polyubiquitination of NLRP3 by the E3 ligase MARCH7. This modification leads to the aggregation of NLRP3 and its subsequent degradation via an autophagic pathway. Dopamine therefore acts as a brake on NLRP3 to limit inflammation.

$L A D$

Cell 160, 62-73(2015)

\section{Healing the CNS}

T cells serve both neurodegenerative roles and neuroprotective roles in the central nervous system (CNS). In the Journal of Clinical Investigation, Kipnis and colleagues characterize the mechanism of $\mathrm{T}$ cell-mediated neuroprotection in two mouse models of CNS injury. CD4 ${ }^{+} \mathrm{T}$ cells are found at sites of CNS injury, and their presence correlates with neuronal protection and functional recovery. The beneficial effect of these $C D 4^{+} T$ cells is dependent on their production of IL-4 but independent of antigen presentation or recognition of any obvious CNS antigen. Instead, the infiltrating $T$ cells are activated to produce IL-4 in a bystander manner by as-yet-unidentified alarmins released from the injured CNS that act via adaptor MyD88-dependent receptors on the $\mathrm{T}$ cells. The IL-4 released by the T cells acts directly on neurons and acts together with other neurotrophic factors to support neuronal growth and repair.

J. Clin. Invest. (20 January 2015) doi:10.1172/JCI76210

\section{Immunological complementation}

Loss-of-function mutations in genes encoding immunologically relevant molecules can often result in an unexpected variation in disease penetrance. In eLife, Virgin and colleagues investigate the cause of this variation by using mice with genetic deficiency in a variety of molecules involved in the positive regulation of inflammatory responses, including HOIL-1, caspase- 1 or IL- 6 alone or caspase-1 plus caspase- 11 . These mutant mice are highly susceptible to certain bacterial infections (such as listeria) yet in some cases (HOIL-1 deficiency) are resistant to others (such as mycobacterium). This susceptibility is dependent mainly on a dysfunctional innate immune response and diminished production of inflammatory cytokines. Chronic infection with the herpesvirus MHV-68 rescues the mutant mice from listerial infection at least in part by normalizing the production of inflammatory cytokines. These findings suggest how the host virome might complement otherwise deficient immune responses and offer insight into the environmental basis of disease penetrance. eLife (20 January 2015) doi:10.7554/eLife.04494

\section{Profiling carbohydrate recognition}

The recognition of bacterial or viral carbohydrate epitopes by antibodies can contribute to immune system-mediated protection against pathogenic microbes. However, the development of potent vaccines against microbial glycans has lagged because of a lack of understanding of how the immune system recognizes carbohydrate structures. In Science Translational Medicine, Schneider et al. describe a systems approach to screening the reactivity of human antibodies to an array of 610 distinct glycan structures. Commerially available intravenous immunglobulin G recognizes several hundred carbohydrate epitopes; the responses are mediated mainly by immunglobulin G2 but not exclusive to this antibody isotype. Notably, glycan structures terminated by galactose, $\mathrm{N}$-acetylgalactosamine or $\mathrm{N}$-acetylglucosamine are prominently recognized, but reactivity to terminal mannose, $\mathrm{N}$-acetylneuraminic acid or the related $\mathrm{N}$-glycolylneuraminic acid is absent or low. These findings indicate that recognition of carbohydrates is selective for the terminal structures of known microbial epitopes or their host glycan-attachment sites and is tolerant of self glycan structures. These findings lend insight into more rational design of glycoconjugate vaccines.

$L A D$

Sci. Transl. Med. 7, 269ra1 (2015)

\section{A20 inhibits the inflammasome}

Mice with deficient expression of the ubiquitin-modifying enzyme A20 spontaneously develop a systemic inflammatory syndrome. In Immunity, $\mathrm{Ma}$ and colleagues show that A20-deficient macrophages secrete the inflammatory cytokine IL-1 $\beta$ in response to stimulation with Toll-like receptor ligands (signal 1) only, while intracellular expression of pro-IL$1 \beta$, but not release of mature IL- $1 \beta$, is observed in wild-type macrophages. The effect is 'rescued' by deletion of components of the NLRP3 inflammasome, such as ASC and caspase-1. Deletion of RIPK3, a kinase involved in the spontaneous activation of NLRP3 in some contexts but independent of A20-mediated regulation of the transcription factor NF- $\kappa \mathrm{B}$, can also 'rescue' this mutant. A20 associates with and inhibits the catalytic activity of a complex containing caspase-1, caspase-8, RIPK1 and RIPK3 and also restricts the Lys63-linked polyubiquitination of pro-IL-1 $\beta$, a modification that promotes its proteolytic cleavage. Thus, A20 suppresses NLRP3 inflammasomes by various different mechanisms. Immunity 42, 55-67 (2015) 